\title{
Effect of Electrical Stimulation on Pain, Grip Strength and Function in Patients with Lateral Epicondylitis
}

\author{
Arul Pragassame $\mathrm{S}^{1}$, Mohan Raj G², Karthik V. ${ }^{3}$ \\ ${ }^{1}$ Department of PMR, RMMCH, Annamalai University, Chidambaram, Tamilnadu, India. ${ }^{2}$ Department of Physiotherapy, \\ Aarupadai Veedu Medical College and Hospital, Puducherry, Tamilnadu, India. \\ ${ }^{3}$ Department of PMR, RMMCH, Annamalai University, Chidambaram, Tamilnadu, India.
}

\section{ABSTRACT}

\section{BACKGROUND}

Lateral epicondylitis (tennis elbow) is a common and often extremely painful musculoskeletal condition. Several non-operative interventions with varying success rates have been described. Systematic reviews suggest that there is no clear and effective cure for pain symptoms in the first six weeks of treatment. There is a strong need for an intervention which is appropriate for patients and ensures effective short-term pain relief without increasing the risk of recurrence. The electrical stimulation using galvanic current is seen to be safe and effective in reducing pain, improving grip strength and promoting functional activities in the treatment of lateral epicondylitis.

\section{METHODS}

Fifteen patients with lateral epicondylitis were selected. Electrical stimulation using galvanic current was applied for a period of 30 seconds at each point 3 times for 10 days. Pain, pain-free grip strength and functional status were measured using the Numerical Pain Rating Scale (NPRS), hand dynamometer and Patient Specific Functional Scale (PSFS). Measurements were taken before and after treatment. Data was subjected to statistical analysis.

\section{RESULTS}

Mean improvement was observed for pain (NPRS), pain free grip strength and functional status (PSFS) $(5.8 \pm 0.9, \mathrm{P}=0.001),(32.6 \pm 2.6, \mathrm{P}=0.001)$ and $(5.2 \pm 1.7$, $\mathrm{P}=0.001$ ) respectively.

\section{CONCLUSIONS}

Application of electrical stimulation using galvanic current was effective in reducing pain, improving grip strength and functional status in patients with lateral epicondylitis.

\section{KEY WORDS}

Electrical Stimulation, Hand Dynamometer, Lateral Epicondylitis. Numerical Pain Rating Scale, Patient Specific Functional Scale
Corresponding Author: Dr, Arul Pragassame $S$, Assistant Professor Department of PMR, RMMCH, Annamalai University, Annamalai Nagar, Chidhambaram-608002, Tamilnadu, India E-mail: arulphysio77@gmail.com

DOI: $10.14260 /$ jemds/2020/370

Financial or Other Competing Interests: None.

How to Cite This Article:

Pragassame SA, Raj MG, Karthik V. Effect of electrical stimulation on pain, grip strength and function in patients with lateral epicondylitis. J. Evolution Med. Dent. Sci. 2020;9(22):1684-1687, DOI: 10.14260/jemds/2020/370

Submission 14-01-2020,

Peer Review 08-05-2020,

Acceptance 16-05-2020,

Published 01-06-2020.

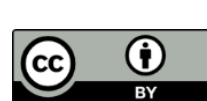




\section{BACKGROUND}

Lateral epicondylitis, also known as tennis elbow, is characterized by pain over the lateral aspect of elbow that is aggravated by wrist extension and direct palpation over the lateral epicondyle of humerus, the radio-humeral joint space or the proximal muscle belly. It is typically work-related or sport-related pain disorder caused by excessive, monotonous, quick, repetitive movements of the wrist. The dominant arm is commonly affected. Repetitive movements and forceful activities were also positively correlated with lateral epicondylitis. ${ }^{1}$

A $50 \%$ prevalence is reported among tennis players older than 30 years of age with a peak age between 35 and 50 years of age. Lateral pain is 5 to 10 times more common than medial pain. ${ }^{2}$ Classic symptoms are pain that is just anterior and distal to the lateral epicondyle associated with wrist and finger extension stress. Wrist flexion and triceps-biceps activity causes no pain. Palpation over the extensor carpi radialis brevis (ECRB) origin causes exquisite pain, while palpation over the lateral epicondyle causes moderate or no pain. ${ }^{3}$ A group of common extensor muscles involved in lateral epicondylitis, the extensor carpi radialis brevis is typically the major contributor to symptoms. The ECRB may be involved in $100 \%$ of cases and the extensor digitorum communis (EDC) is involved in $30 \%$ of cases. ${ }^{4}$

The role of the wrist extensor is to place and stabilize the wrist for finger-involving activities, particularly when making the fist. Activities involving repetitive firm grip, such as playing tennis or hammering, can overwork the wrist extensors, particularly the highly active ECRB. A condition known as tennis elbow or lateral epicondylitis is caused by stress and resulting information on the proximal attachment of the wrist extensors. ${ }^{5}$

Several interventions have been described for the management of lateral epicondylitis, including NSAIDs, corticosteroid injection, Muscle Strengthening and stretching exercise, Cryotherapy, use of orthotic device, Taping techniques, Manipulative technique, Ultrasound, TENS, LASER, ESWT, Electromagnetic field and Ionization. Although many studies have conducted conservative treatment of this condition, there is no agreement on the most effective management strategy. ${ }^{6}$

According to Bissert et al, a systematic randomized controlled trial was conducted on the effectiveness of various conventional treatment on tennis elbow. They concluded that conventional treatment did not have a long-term effect on symptoms. $^{7}$ Chop WM proposed as an alternative pathophysiology that some of the symptoms of lateral epicondylitis could be attributed to tender points in the lateral elbow, overuse of forceful arm and wrist activities. ${ }^{8}$ Chop WM attributes the formation of such tender points, is due to increased sensitivities of the neural receptor in the muscle, and is related to connective tissue in response to overuse. Localized tender points in the lateral elbow area may cause pain when palpated, stretched or overloaded. Electrical stimulation was used for trigger point treatment without any known anti-inflammatory offsets. ${ }^{8}$ This study, proposed solely for trigger point treatment, could provide a variable alternative treatment for lateral epicondylitis. The purpose of this study is to examine the effect of electrical stimulation using galvanic current on symptoms of lateral epicondylitis.

\section{METHODS}

The present study was an interventional study conducted in the outpatient Department of Physical Medicine and Rehabilitation (PMR), RMMCH, Annamalai University, Tamil Nadu, India, during September, October and November 2019. The study was approved by the Departmental Research Committee (PMR/DRC-8/2019)

During the stipulated period, the sample size was selected using a convenient sampling method. Fifteen patients with lateral epicondylitis were selected. The criteria for the inclusion in the study were (1) Clinically diagnosed cases of lateral epicondylitis; (2) Both sides (right and left) included; (3) Age group over 25 years of age; (4) Both male and female. The main exclusion criteria were (1) Radiculopathy from cervical spondylitis; (2) recent steroid injection at the site; (3) Cardiovascular disease; (4) Uncontrolled diabetes; (5) Previous trauma/surgery in the region of lateral epicondylitis.

\section{Study Procedure}

Patients were informed of the purpose, study method, and informed consent obtained individually for participation after screening for selection criteria. First, the subject was assessed for pain, grip strength, and functional activity using the Numeric Pain Rating Scale (NPRS) ${ }^{9}$, Hand Dynamometer ${ }^{10}$ and the Patient Specific Functional Scale (PSFS). ${ }^{11}$ The patients were treated with electrical stimulation using galvanic current over the palpated tender point for 30 seconds on each tender point 3 times for 10 days. Following completion of treatment, the same evaluation was performed to evaluate the effectiveness of electrical stimulation using galvanic current for lateral epicondylitis. Pre-and posttreatment values were then furnished statistically.

\section{Outcome Measures}

Numerical Pain Rating Scale- The NPRS is a subjective measure that allows individuals to evaluate their pain at an eleven-point numerical scale. The scale ranges of 0 (no pain at all) to 10 (worst possible pain). Patients were asked to mark a line point referring to their perceived quantity of pain during the evaluation'.

Pain Free Grip Strength- Pain free grip strength is a measure of the grip force needed to cause the onset of pain. Pain free grip force was used as an outcome measure because it reflects the degree of impairment associated with lateral epicondylitis. Hand dynamometer was used to measure the grip strength of the affected arm. It is used to measure painfree grip force over 3 repetitions with a rest interval of 30 seconds. The test was performed with the participants arm placed by his/her side with elbow extended and forearm pronated.10 
Patient Specific Functional Scale- The PSFS is a self-reported, designed to assess functional changes, particularly in patients with musculoskeletal disorders. The PSFS enables patients to report their functional status as a clinical outcome measure at the baseline and at a follow-up session to determine whether a significant change in functional status has occurred ${ }^{11}$.

\section{Treatment Procedure}

Application of Electrical Stimulation (Galvanic Current)During treatment, the patient was seated with the affected arm resting on the table. After palpation of the painful area of the lateral epicondyle while the subject held a ground electrode in his or her affected hand, the therapist searched the tender spot area of the lateral epicondyle with the tip of his finger. When the tender points were located, electrical stimulation using galvanic current was applied for a $30 \mathrm{sec}$ duration on each tender point 3 times for 10 days. The stimulation intensity was adjusted to the subject's pain tolerance level. During each therapy session, all identified points were stimulated three times. During the treatment period, the subject could hear a beeping tone signifying that a stimulus was being delivered.

\section{Dosage \\ Intensity- Up to tolerable level.}

Mode- Interrupted.

Treatment Duration- 90 Sec.

Patient Position- Sitting position, Shoulder: Abducted, Elbow: Flexed 90, Forearm: Pronated, Wrist: Neutral Position.

\section{Statistical Analysis}

Statistical analysis of the study was carried out using the "SxStatistical Package" to test the hypothesis that treatment had a significant difference between before and after treatment. The test for the different samples (paired t-test) was carried out. The level of significance $\alpha=0.05$. The data were submitted in a tabular format.

\section{RESULTS}

The table 1 shows the mean value for pain before treatment is $7.8 \pm 1.2$ and the mean value for pain after treatment is $2.0 \pm 0.7$. It shows the statistically significant at the $P=0.001$ level with a ' $t$ ' value of 30.32 . Therefore, hypothesis is rejected and hence there is significant difference between pre and post treatment.

The table 2 shows the mean value for grip strength before treatment is $10.26 \pm 3.5$ and the mean value for grip strength after treatment is $42.86 \pm 1.7$. It shows the statistically significant at the $\mathrm{P}=0.001$ level with a ' $\mathrm{t}$ ' value of -16.75 . Therefore, hypothesis is rejected and hence there is significant difference between pre and post treatment.
The table 3 shows the mean value for PSFS before treatment is $2.34 \pm 2.1$ and the mean value for PSFS after treatment is $7.54 \pm 1.4$. It shows the statistically significant at the $\mathrm{P}=0.001$ level with a ' $\mathrm{t}$ ' value of 36.76 . Therefore, hypothesis is rejected and hence there is significant difference between pre and post treatment.

\begin{tabular}{|ccccc|}
\hline No. of Subjects & NPRS & Mean \pm SD & 'T' Value & P Value \\
\multirow{2}{*}{15} & Pre -Treatment & $7.8 \pm 1.2$ & 30.32 & 0.001 \\
& Post -Treatment & $2.0 \pm 0.7$ & 30. \\
\hline \multicolumn{5}{|c}{ Table 1. NPRS (Pre- and Post-Treatment Values) } \\
\hline
\end{tabular}

\begin{tabular}{|ccccc|}
\hline No. of Subjects & Pain Free Grip Strength (Kg) & Mean \pm SD & 'T' Value & P Value \\
15 & Pre -Treatment & $10.26 \pm 3.5$ & 16.75 & 0.001 \\
\hline \multicolumn{4}{|c|}{ Table 2. Pain Free Grip Strength (Pre- and Post-Treatment Values) } \\
\hline
\end{tabular}

\begin{tabular}{|ccccc|}
\hline No. of Subjects & PSFS & Mean \pm SD & 'T' Value & P Value \\
\multirow{2}{*}{15} & Pre -Treatment & $2.34 \pm 2.1$ & 36.76 & 0.001 \\
& Post -Treatment & $7.54 \pm 1.4$ & \\
\hline \multicolumn{5}{|c}{ Table 3. PSFS (Pre- and Post-Treatment Values) } \\
\hline
\end{tabular}

\section{DISCUSSION}

The objective of this study is to investigate the effect of electrical stimulation using galvanic current on pain, grip strength, and functional activity in subjects with lateral epicondylitis. It is an interventional study extended over a period of three months in the department of Physical Medicine and Rehabilitation, RMMCH, Annamalai University.

The results of this study indicated that the treatment of tender spots over the lateral epicondylitis with lowfrequency galvanic stimulation could clinically improve pain, grip strength and functional activity in subjects with lateral epicondylitis.

These findings could support the hypothesis that the symptoms of lateral epicondylitis might be due to active tender points rather than to inflammatory reactions. The results of this study showed a significant reduction $(\mathrm{p}=0.000)$ in pain intensity in patients with lateral epicondylitis.

According to "Spadoni et al," a reduction in pain greater than 3.0 points in the NPRS is considered clinically significant. Therefore, our findings of average pain value with a decrease in pain intensity after treatment are much less than the before treatment. Data related to individual subjects revealed that $100 \%$ of cases in the treatment group showed a reduction of more than 3.0 points in pain intensity after treatment. ${ }^{12}$

The significant improvement in grip strength found in this study could be related to decreased pain and improved functional activity. Pain and tenderness in the wrist extensor muscles working as wrist stabilizers in synergy with the finger flexor muscles "could affect the grip strength" through electrical stimulation increases in collagen synthesis, reduction in pain by closing pain gate, reduction in muscle spasm, improvement in blood circulation and breaking the adhesion formation. As a result of improved pain, and grip strength, there will be significant improvement in functional activities and the use of the affected arm in daily activities. The patient specific functional scale provides a functional improvement in the study. ${ }^{13}$ 
The results of this study suggest that the effect of electrical stimulation on pain control have a neurophysiological rather than psychological basis.

\section{Limitations and Future Directions}

Small sample size is a limitation of this study. This study may be analyzed by comparing it with other electrotherapeutic approaches.

\section{CONCLUSIONS}

There is significant improvement with regard to pain, grip strength, and functional performance, in patients treated with electrical stimulation. It can therefore be concluded that electrical stimulation using galvanic current is effective in the treatment of subjects suffering from lateral epicondylitis.

\section{ACKNOWLEDGEMENT}

The authors would like to thank Prof. V. K Mohandas Kurup, former Professor and Head of the Department of Physical Medicine and Rehabilitation at Raja Muthiah Medical College and Hospital, Annamalai University for his guidance and encouragement.

\section{REFERENCES}

[1] Stasinopoulos D, Stasinopoulou K, Johnson MI. An exercise programme for the management of lateral elbow tendinopathy. Br J Sports Med 2005;39(12):944-7.

[2] Nourbakhsh MR, Fearon FJ. An alternative approach to treating lateral epicondylitis. A randomized, placebocontrolled, double-blinded study. Clin Rehabil 2008;22(7):601-9.
[3] Reider B. Sports medicine. $2^{\text {nd }}$ edn. Pennsylvania: W.B. Saunders Company, 1996.

[4] Hall CM, Brody LT. Therapeutic exercises. $2^{\text {nd }}$ edon. New York: Lippincott Williams \& Wilkins 1999.

[5] Neumann DA. Kinesiology of the musculoskeletal system. $3^{\text {rd }}$ edn. Philadelphia: Mosby 1999.

[6] Labelle H, Guibert R, Joncas J, et al. Lack of scientific evidence for the treatment of lateral epicondylitis of the elbow. An attempted meta-analysis. J Bone Joint Surg Br 1992;74(5):646-51.

[7] Bisset L, Paungmali A, Vicenzino B, et al. A systematic review and meta-analysis of clinical trials on physical interventions for lateral epicondylalgia. Br J Sports Med 2005;39(7):411-22.

[8] Chop Jr WM. Tennis elbow. Postgraduate Medicine 1989;86(5):301-8.

[9] Alghadir AH, Anwer S, Iqbal A, et al. Test-retest reliability, validity and minimum detectable change of visual analog, numerical rating and verbal rating scales for measurement of osteoarthritis knee pain. J Pain Res 2018;11:851-6.

[10] Wuori JL, Overend TJ, Kramer JF, et al. Strength and pain measures associated with lateral epicondylitis bracing. Arch Phys Med Rehabil 1998;79(7):832-7.

[11] Hall AM, Maher CG, Latimer J, et al. The patient-specific functional scale is more responsive than the Roland Morris disability questionnaire when the activity limitation is low. Eur Spine J 2011;20(1):79-86.

[12] Spadoni GF, Stratford PW, Solomon PE, et al. The evaluation of change in pain intensity: a comparison of the P4 and single-item numeric pain rating scales. J Orthop Sports Phys Ther 2004;34(4):187-93.

[13] Nourbakhsh MZ, Fearon FJ. An alternative approach to treating lateral epicondylitis. A randomized, placebocontrolled, double-blinded study. Clinical Rehabilitation 2008;22(7):601-9. 